\title{
Identification and fine mapping of a linear B cell epitope of human vimentin.
}

\author{
Dam, Catharina Essendrup; Houen, Gunnar; Hansen, Paul R.; Trier, Nicole H.
}

\section{Published in:}

Scandinavian Journal of Clinical \& Laboratory Investigation

Link to article, DOI:

10.3109/00365513.2014.908474

Publication date:

2014

Document Version

Publisher's PDF, also known as Version of record

Link back to DTU Orbit

Citation (APA):

Dam, C. E., Houen, G., Hansen, P. R., \& Trier, N. H. (2014). Identification and fine mapping of a linear B cell epitope of human vimentin. Scandinavian Journal of Clinical \& Laboratory Investigation, 74(6), 506-514. https://doi.org/10.3109/00365513.2014.908474

\section{General rights}

Copyright and moral rights for the publications made accessible in the public portal are retained by the authors and/or other copyright owners and it is a condition of accessing publications that users recognise and abide by the legal requirements associated with these rights.

- Users may download and print one copy of any publication from the public portal for the purpose of private study or research.

- You may not further distribute the material or use it for any profit-making activity or commercial gain

- You may freely distribute the URL identifying the publication in the public portal 


\title{
Identification and fine mapping of a linear B cell epitope of human vimentin
}

\author{
CATHARINA E. DAM ${ }^{1,2}$, GUNNAR HOUEN ${ }^{2}$, PAUL R. HANSEN ${ }^{3}$ \\ \& NICOLE H. TRIER ${ }^{2}$ \\ ${ }^{1}$ Department of Systems Biology, Technical University of Denmark, Kongens Lyngby, Denmark, \\ ${ }^{2}$ Department of Clinical Biochemistry, Immunology E Genetics, Statens Serum Institut, Copenhagen, Denmark, \\ and ${ }^{3}$ Department of Drug Design and Pharmacology, Faculty of Health and Medical Sciences, \\ University of Copenhagen, Copenhagen, Denmark
}

\begin{abstract}
Knowledge about antibody-antigen interactions is important for the understanding of the immune system mechanisms and for supporting development of drugs and biomarkers. A tool for identification of these antigenic epitopes of specific antibodies is epitope mapping. In this study, a modified enzyme-linked immunosorbent assay was applied for epitope mapping of a mouse monoclonal vimentin antibody using overlapping resin-bound peptides covering the entire vimentin protein. The minimal epitope required for binding was identified as the LDSLPLVD sequence using $N$ - and $C$-terminally truncated peptides. The peptide sequence LDSLPLVDTH was identified as the complete epitope, corresponding to amino acids 428-437 in the $C$-terminal end of the human vimentin protein. Alanine scanning and functionality scanning applying substituted peptides were used to identify amino acids essential for antibody reactivity. In particular, the two aspartate residues were found to be essential for antibody reactivity since these amino acids could not be substituted without a reduction in antibody reactivity. The majority of the remaining amino acids could be substituted without reducing antibody reactivity notably. These results confirm that charged amino acids are essential for antibody reactivity and that the vimentin antibody is dependent on side-chain interactions in combination with backbone interactions.
\end{abstract}

Key Words: Epitope mapping, monoclonal antibody, solid-phase peptide synthesis, synthetic resin-bound peptides

Abbreviations: AP, Alkaline phosphatase; ELISA, Enzyme-linked immunosorbent assay; FITC, Fluorescein isothiocyanate; Fmoc, 9-fluorenylmethoxycarbonyl; HOAt, 1-hydroxy-7-aza-benzotriazole; MBL, Mannan-binding lectin; NMP, $N$-methyl-2-pyrrolidone; PBS, Phosphate buffered saline; RT, Room temperature; SPPS, Solid-phase peptide synthesis; TFA, trifluoroacetic acid; TTN, Tris-Tween-NaCl

\section{Introduction}

The antigen-antibody interaction is important for autoimmune responses and immune responses against pathogens. The interface between an antibody and its cognate antigen is usually complementary with regard to structure and size, making the interaction highly specific. This interaction involves several non-covalent forces [1,2]. Antibodies recognize different motifs in antigens such as extended chains, $\alpha$-helices and $\beta$-turns, of which the latter are the most common motifs observed in B cell epitopes. Antibodies may interact with the amino acid side chains of the epitope, the backbone atoms or a combination of the two [3-5]. An antibody is often highly specific for its epitope, which implies that several amino acid side chains cannot be replaced without losing antibody binding [4].

Antibodies are capable of recognizing linear and conformational B cell epitopes. These epitopes are often 10-25 amino acids long, however, some variation in epitope length has been observed $[2,6,7]$. Epitopes identified tend to be highly accessible on the surface of the antigen, hence polar and charged amino acids are often found in epitopes. These preferences are most likely due to that polar and charged amino acid residues form a variety of interactions with the antibodies $[6,8]$. Several studies have 
suggested that epitopes have a flexible backbone and are located in flexible regions that protrude from the protein surface. These properties allow the epitope to adopt a conformation that is complementary to the structure of the antigen-binding site of the antibody $[4,6]$.

Identification of epitopes, also known as epitope mapping, provides valuable information about the interaction between an antibody and its cognate antigen. The importance of each amino acid in the epitope for antibody binding can be determined by epitope mapping. Several methods are used for mapping of epitopes, depending on whether linear or conformational epitopes are mapped. X-ray crystallography is applied for identification of both linear and conformational epitopes, however, this approach is time consuming and requires large amounts of the antigen and antibody to obtain crystals of good quality. Linear epitopes can be mapped by a variety of strategies, e.g. enzyme-linked immunosorbent assay (ELISA). Linear epitopes have been studied more extensively than conformational epitopes since they are easier to identify and are used for many applications $[3,9]$. An efficient strategy for identifying linear epitopes by ELISA is to use overlapping synthetic peptides covering the entire antigen. This method, also referred to as peptide scan, was originally described by Geysen et al. [10] in 1984, who determined a 7-mer epitope of an antibody specific for a viral coat protein, which contained two Leu residues important for antibody binding. Synthetic peptides have been used for epitope mapping in several studies due to their many advantages. They are very pure, easily synthesized and chemically or posttranslationally modified amino acids can be incorporated [11] Synthetic resin-bound peptides have been used in a few studies for mapping and characterization of $B$ cell epitopes [12-14]. The advantage of using resinbound peptides is that the peptides are flexible since only one amino acid is attached to the solid support. Furthermore, using resin-bound peptides instead of free peptides, coating steps in ELISA are avoided, making this method faster [14,15]. Resin-bound peptides were originally used for epitope mapping in 1985 by Paterson [12] who identified two epitopes in rat cytochrome c. Synthetic resin-bound peptides have also been used in a study by Tian et al. [13] for characterization of the 6-mer ELDKWA epitope from the gp41 protein of HIV-1 using monoclonal antibodies. In the study by Tian et al. [13], charged amino acids, such as Asp, and the hydrophobic amino acid Leu were shown to be important for antibody binding. The 9-mer peptide LELDKWASL was identified as the complete epitope attaining maximal affinity [13]. These types of epitope mapping studies provide knowledge about the location of epitopes within an antigen and are important for the development of diagnostic kits, design of vaccines and immunotherapy. Furthermore, determination and characterization of epitopes are also important for understanding the pathophysiology of many diseases, including autoimmune diseases $[6,16]$.

Several epitopes have been described in multiple proteins, such as vimentin, which is a protein subunit of intermediate filaments found in mesenchymal cells $[17,18]$. Vimentin is a part of the cytoskeleton and is involved in stabilization of the molecular network. Moreover, vimentin functions in organelle transport, cell migration and proliferation. The broad range of physiological functions of vimentin is also underlined by the intracellular and extracellular localization as well as the possibility of modifications such as phosphorylation and citrullination [19-21]. Antibodies targeting modified vimentin have been suggested to have a role in the autoimmune disease rheumatoid arthritis and are used as a marker for diagnosis of the disease [22,23].

In this study, the epitope of a mouse monoclonal vimentin antibody was fine mapped using overlapping synthetic resin-bound vimentin peptides. The identified epitope of the vimentin antibody was further characterized using both truncated peptides and substituted peptides. Moreover, the substituted peptides were applied to examine which kinds of modifications the antibody was able to tolerate.

\section{Materials and methods}

\section{Reagents}

Overlapping resin-bound vimentin peptides, $C$ terminally truncated resin-bound peptides and substituted peptides were purchased from Schafer-N. $\mathrm{N}$-terminally truncated vimentin peptides were synthesized by 9-fluorenylmethoxycarbonyl (Fmoc)based solid-phase peptide synthesis (SPPS) as described below. Tris-Tween-NaCl (TTN) buffer (0.0 5M Tris, $0.3 \mathrm{M} \mathrm{NaCl}, 1 \%$ Tween 20 , pH 7.4), alkaline phosphatase (AP) substrate buffer (1 M diethanolamine, $0.5 \mathrm{mM} \mathrm{MgCl}{ }_{2}, \mathrm{pH}$ 9.8), phosphate buffered saline (PBS, $\mathrm{pH}$ 7.2), glycerol and mouse monoclonal human mannan-binding lectin (MBL) antibody (HYB 131-01) were from Statens Serum Institut. AP-conjugated anti-mouse IgG (A3688), paranitrophenylphosphate, $N, N^{\prime}$-diisopropylcarbodiimide, piperidine, trifluoroacetic acid (TFA), ethanol and ether were purchased from Sigma-Aldrich. Mouse monoclonal human vimentin antibody was purchased from Biosite (AMB-7100). TentaGel S NH resin was purchased from RAPP Polymere $\mathrm{GmbH}$ (S30902). Fmoc-protected amino acids and $N$ methyl-2-pyrrolidone (NMP) were purchased from Iris Biotech GmbH. 1-hydroxy-7-aza-benzotriazole (HOAt) was purchased from GL Biochem. Triisopropylsilane was purchased from Merck; a 13-well HEp-2 slides ANA Test system HEp-2 substrate was purchased from Immuno Concepts (REF2013). Fluorescein isothiocyanate (FITC)-conjugated antimouse IgG was purchased from Dako (F0261). 


\section{Peptides}

Overlapping resin-bound vimentin peptides, spanning the complete human vimentin sequence (Accession number: P08670), were constructed as 20-mer peptides with a 10 amino acid overlap. In total 46 peptides were generated (See Supplementary Appendix to be found online at http://informahealthcare.com/ doi/abs/10.3109/00365513.2014.908474).

\section{Indirect immunofluorescence}

Slides with HEp-2 cells were incubated with primary antibody (vimentin antibody or control MBL antibody) diluted in PBS (1:160-1:2560). The slides were incubated in a humidity chamber at room temperature (RT) for $30 \mathrm{~min}$. The $\mathrm{HEp}-2$ slides were then washed 5 times with PBS followed by $10 \mathrm{~min}$ incubation at RT in PBS. FITC-conjugated anti-mouse IgG diluted in PBS (1:40) was added to the slides and incubated in a humidity chamber at RT for 30 min followed by a PBS wash for $10 \mathrm{~min}$. After wiping the slides, glycerol was added as a mounting medium and the wells were covered with a cover glass. The slides were analyzed using an Olympus DP72 fluorescence microscope (Olympus, PA, USA).

\section{Solid-phase peptide synthesis of $\mathrm{N}$-terminally truncated peptides}

$N$-terminally truncated resin-bound peptides, using the sequence RETNLDSLPLVDTH from vimentin as template, were synthesized by traditional Fmoc-based SPPS as described previously [24]. Briefly, $20 \mathrm{mg}$ resin was removed from the resin pool after coupling of each amino acid except for the first three amino acids. $12 \mathrm{~N}$-terminally truncated resin-bound peptides were synthesized in total. The peptides were synthesized in a syringe using TentaGel $\mathrm{S} \mathrm{NH} \mathrm{NH}_{2}$ resin $(270 \mathrm{mg}$, loading capacity $0.31 \mathrm{mmol} / \mathrm{g}$ ). Amino acid activation and coupling was conducted using three-fold excess of Fmoc-protected amino acids, HOAt and $N, N^{\prime}-$ diisopropylcarbodiimide. All amino acids were double coupled for at least $2.5 \mathrm{~h}$ in total at RT with a NMP wash between the two couplings. Following coupling, the resin was rinsed 7 times with NMP and deprotection was conducted using $20 \%$ piperidine in NMP 3 times for $17 \mathrm{~min}$ in total. Following synthesis of the peptides, the resin was washed twice with ethanol and ether, respectively. The side chain protection groups were removed using a 5\% triisopropylsilane and 95\% TFA solution for $2 \mathrm{~h}$ at RT. The resin-bound peptides were rinsed twice with TFA and three times with ether and the remaining ether was evaporated over-night.

\section{Modified enzyme-linked immunosorbent assay}

The resin-bound peptides $(3.3 \mathrm{mg} / \mathrm{mL}$ resin) were added to a 96-well multiscreen HTS filter plate (Millipore, Copenhagen, Denmark) and washed with TTN buffer using a multiscreen vacuum manifold (Millipore, Copenhagen, Denmark). The wells were blocked with TTN buffer for $20 \mathrm{~min}$ at RT. Primary mouse monoclonal antibody directed against vimentin or MBL $(1: 1000)$ was added and the plate was incubated for 1 hour at RT on a shaking table. Secondary AP-conjugated anti-mouse IgG $(1: 1000)$ was added and the plate was incubated for $1 \mathrm{~h}$ at RT on a shaking table. Both incubations with antibodies were followed by 4 washes with TTN buffer. For quantification of bound antibodies, paranitrophenylphosphate $(1 \mathrm{mg} / \mathrm{mL})$ diluted in AP substrate buffer was used. The buffer was transferred to a Maxisorp microtitre plate (Nunc, Roskilde, Denmark) and the absorbance was determined at $405 \mathrm{~nm}$, with background subtraction at $650 \mathrm{~nm}$, using a Thermomax microtitre plate reader (Molecular Devices, Menlo Park, CA, USA).

\section{Results \\ Confirmation of antibody specificity}

The monoclonal antibody directed against vimentin was tested by indirect immunofluorescence to check the specificity of the antibody. The reactivity of the monoclonal vimentin antibody to HEp-2 cells revealed a typical vimentin pattern $[25,26]$, confirming the specificity for vimentin (Figure 1). No patterns were detected with the control antibody directed against MBL.

\section{Screening of vimentin peptides with a monoclonal vimentin antibody}

To identify a potential epitope within the vimentin protein, the vimentin antibody was screened for reactivity to 46 overlapping resin-bound vimentin peptides by modified ELISA. A monoclonal control antibody of irrelevant specificity directed against MBL was used as a negative control.

As illustrated in Figure 2, the monoclonal vimentin antibody recognized two peptides, peptide 2 (YRRMFGGPGTASRPSSSRSY, 11-30) and peptide 43 (LNLRETNLDSLPLVDTHSKR, 421-440). Moreover, the control antibody of irrelevant specificity was found to recognize peptide 2 as well, indicating that antibody reactivity to peptide 2 was nonspecific. Thus, specific reactivity was only obtained to peptide 43 , suggesting that the epitope of the vimentin antibody is located within peptide 43. Furthermore, no reactivity was observed with peptides 42 and 44, indicating that the epitope spans the overlap of these two peptides. 


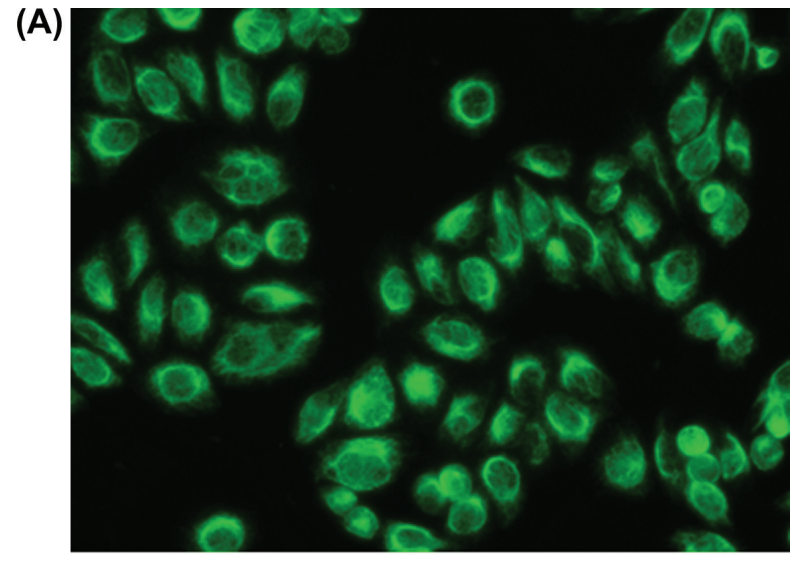

(B)

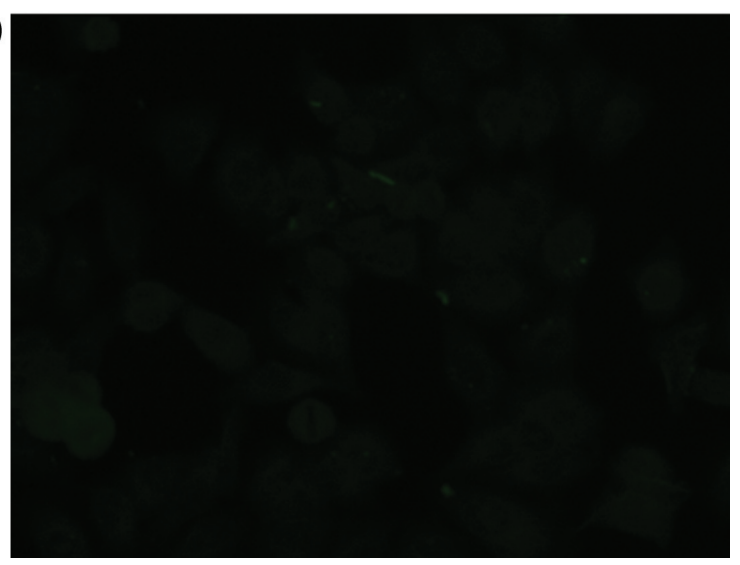

Figure 1. Reactivity of antibodies directed against vimentin and mannan-binding lectin to HEp-2 cells analyzed by indirect immunofluorescence. (A) Vimentin antibody, diluted 1:2560. (B) Mannan-binding lectin antibody, diluted 1:160. This Figure is reproduced in colour in the online version of The Scandinavian fournal of Clinical \& Laboratory Investigation.

Identification of terminal epitope borders using $\mathrm{N}$ - and C-terminally truncated resin-bound peptides

In order to determine the minimal epitope essential for antibody reactivity within peptide $43, N$-terminally truncated resin-bound peptides, using peptide 43 as template, were synthesized by Fmoc-based SPPS and the vimentin antibody was tested for reactivity to the peptides by modified ELISA. As shown in Figure $3 \mathrm{~A}$, no interaction was observed between the vimentin antibody and the seven shortest peptides (DTHDSLPVDTH). The peptide LDSLPLVDTH was the shortest resin-bound peptide found to interact with the vimentin antibody. This result indicated that the $N$-terminal Leu of LDSLPLVDTH was important for antibody reactivity. The interaction between the antibody and the peptides longer than LDSLPLVDTH showed almost no further increase in antibody reactivity compared to the control (peptide 43).

Based on these findings, antibody reactivity to $C$-terminally truncated vimentin peptides was studied by modified ELISA using LDSLPLVDTH as a template. As seen in Figure 3B, only two of the $C$-terminally truncated peptides were recognized by the vimentin antibody. The antibody showed no reactivity to the five shortest peptides (LDS-LDSLPLV). LDSLPLVD was the shortest peptide recognized by the vimentin antibody, indicating that the $C$-terminal Asp was important for antibody reactivity. To sum up, the terminal truncation studies suggested that LDSLPLVD was the minimal epitope of the vimentin antibody.

\section{Screening of epitope candidates for identification of the complete epitope}

Based on the results from the initial screenings of $\mathrm{N}$ - and $\mathrm{C}$-terminally truncated vimentin peptides, the complete epitope of the vimentin antibody was identified by screening selected epitope candidates using modified ELISA.

As demonstrated in Figure 4, LDSLPLVD was the shortest peptide found to interact with the antibody,

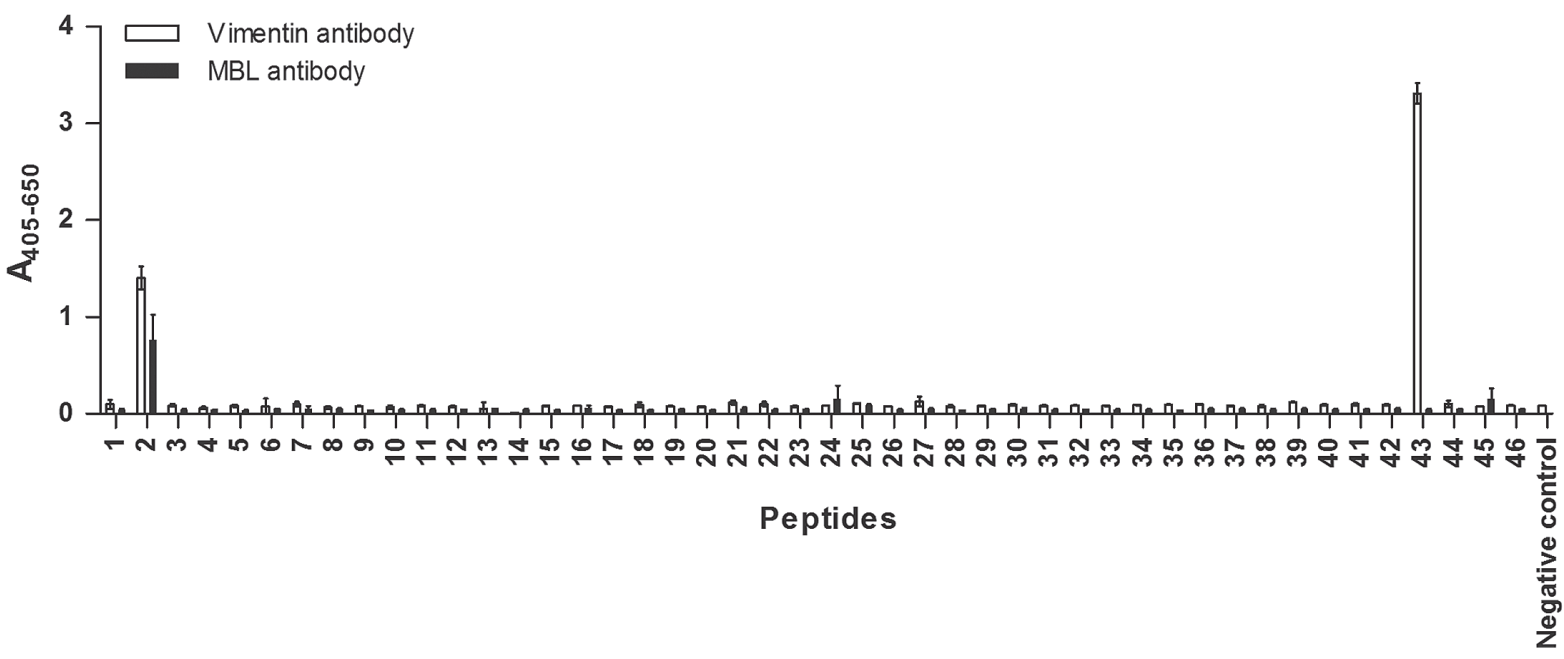

Figure 2. Reactivity of a mouse monoclonal vimentin antibody and MBL antibody to 46 overlapping resin-bound vimentin peptides analyzed by modified ELISA. Negative control: peptide of irrelevant specificity was used. The sequence for the negative control peptide is shown in the Supplementary Appendix to be found online at http://informahealthcare.com/doi/abs/10.3109/00365513.2014.908474. Antibody reactivity to each peptide was analyzed in duplicate. Relative to controls specific antibody reactivity was found to peptide 43. 

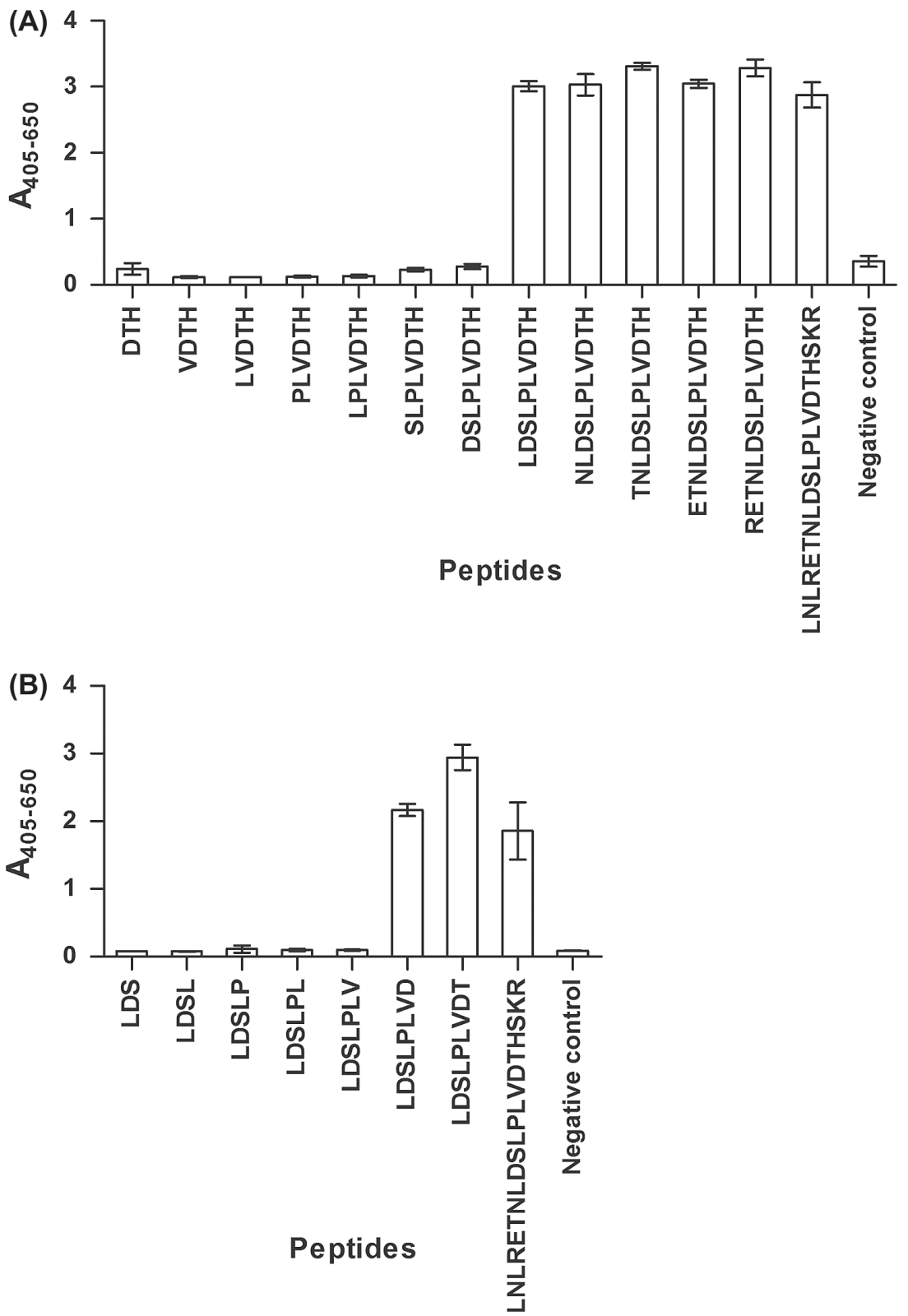

Figure 3. (A) Reactivity of the vimentin antibody to $N$-terminally truncated resin-bound vimentin peptides and (B) $C$-terminally truncated resin-bound vimentin peptides measured by modified ELISA. Peptide 43 (LNLRETNLDSLPLVDTHSKR) was used as a positive control. Uncoated resin was used as a negative control. Antibody reactivity to each peptide was analyzed in duplicate.

supporting the finding that this peptide was the minimal epitope of the vimentin antibody. A small increase in antibody reactivity (approx. 20\%) was observed from LDSLPLVD to LDSLPLVDT and similarly from LDSLPLVDT to LDSLPLVDTH, indicating that the $C$-terminal $\mathrm{Thr}$ and His were also important for antibody reactivity. These results suggested that the amino acid sequence LDSLPLVDTH constituted the complete epitope of the vimentin antibody.

\section{Analysis of the vimentin antibody epitope by alanine scanning}

In order to identify the amino acids of the epitope that were essential for antibody binding, an Ala scanning was performed by modified ELISA. The peptide
N1LDSLPLVDTH11 (numbers indicate position of amino acids in the peptide) was used as template for generation of Ala-substituted peptides.

As illustrated in Figure 5, antibody reactivity to the peptides, where Asp3 and Asp9 were substituted with Ala, was almost completely reduced (89-97\% reduction), compared to the control peptide NLDSLPLVDTH. This indicated that these amino acids were essential for reactivity with the vimentin antibody. Furthermore, a notable reduction in antibody reactivity was observed when Leu2 and Leu5 were substituted with Ala, suggesting that they also influenced antibody binding. Substitution of the remaining amino acids with Ala yielded nearly no difference in reactivity compared to the control peptide, suggesting that the side chains of 


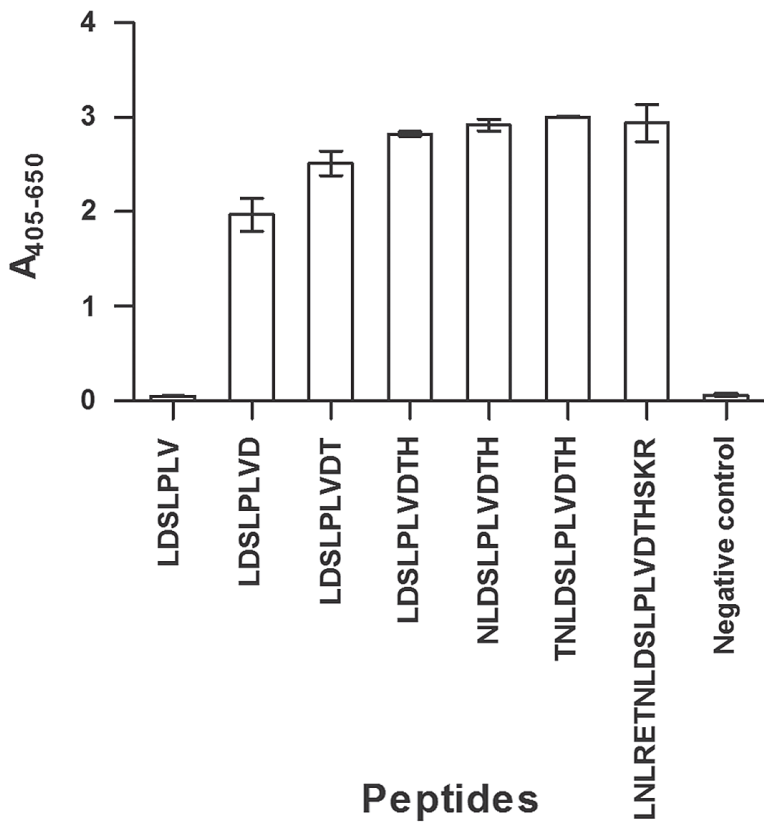

Figure 4. Reactivity of the vimentin antibody to resin-bound vimentin epitope candidates analyzed by modified ELISA. Peptide 43 (LNLRETNLDSLPLVDTHSKR) was used as a positive control. The peptide LDSLPLV and uncoated resin were used negative controls. Antibody reactivity to each peptide was analyzed in duplicate.

these amino acids were not involved in the binding to the vimentin antibody.

\section{Analysis of vimentin antibody reactivity to functional-substituted peptides}

In order to examine which kind of substitutions that were tolerated by the vimentin antibody in each position of the identified epitope, a functionality scanning was performed, in which each amino acid in the peptide sequence was substituted with different amino acids, mainly with amino acids with similar

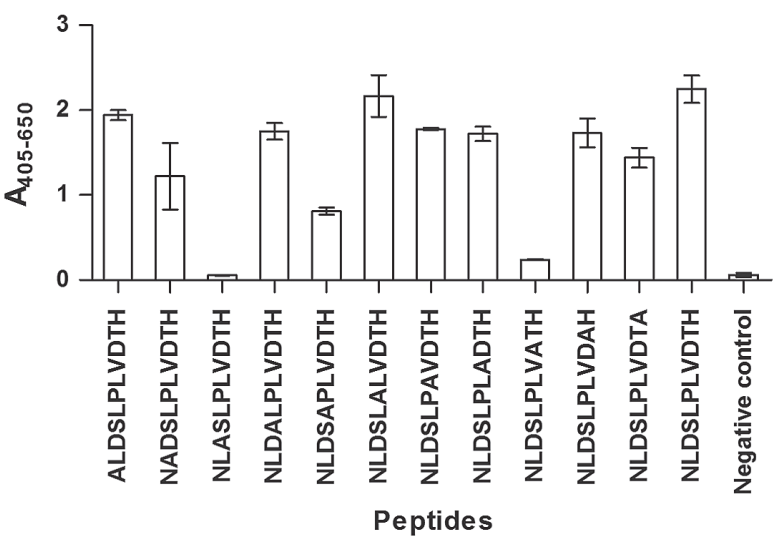

Figure 5. Reactivity of the vimentin antibody to Ala-substituted resin-bound vimentin peptides analyzed by modified ELISA. The peptide NLDSLPLVDTH was used as the positive control peptide with no substitutions. Uncoated resin was used as a negative control. Antibody reactivity to each peptide was analyzed in duplicate. side chain functionality or size. The influence of these varying substitutions on antibody reactivity to the NLDSLPLVDTH peptide was analyzed by a modified ELISA.

Figure 6 illustrates antibody reactivity to substituted peptides. As illustrated, when substituting Asp3 with either Glu or Asn, antibody reactivity was reduced by approx. $100 \%$ compared to the control peptide NLDSLPLVDTH. Substitution of Asp9 with Asn resulted in approximately $80 \%$ reduction compared to the control peptide. When Leu5 was substituted with Ile or Val a reduction of approximately $50 \%$ and $70 \%$, respectively, was observed. The substitution of Pro6 with Gly resulted in a very large reduction, almost $100 \%$ compared to the control peptide.

These results suggested that Asp3, Asp9, Leu5 and Pro6 were important for antibody reactivity. In contrast, substitution of the amino acids in the remaining positions only showed a slight reduction in reactivity, indicating that these specific amino acids were not important for antibody binding.

\section{Discussion}

In this study, the reactivity of a monoclonal vimentin antibody to overlapping resin-bound peptides, covering the entire human vimentin protein sequence, was analyzed in order to locate and characterize the epitope. Peptide 43, corresponding to amino acids LNLRETNLDSLPLVDTHSKR (position 421-440) was the only peptide specifically recognized by the antibody, as illustrated in Figure 2. The epitope was assumed to involve the middle of the 20-mer peptide, since only one peptide was recognized in a specific manner and the two neighboring peptides showed no reactivity. Based on these findings, $N$ - and $C$-terminally truncated peptides were generated and analyzed for antibody reactivity, identifying the peptide LDSLPLVD as the minimal epitope. These findings indicated that the $N$-terminal Leu and the $C$-terminal Asp were important for antibody recognition (Figure 3). Further studies identified the amino acid sequence LDSLPLVDTH as the complete epitope, suggesting that the $C$-terminal Thr and His were important but not essential for antibody binding (Figure 4).

The identified vimentin epitope was found to be located in the tail region of vimentin. As no crystal structure, covering this particular region of human vimentin, is available, the secondary structure of this region has not been determined. Nevertheless, a study by Hess et al. [27], using site directed spin labeling and electron paramagnetic resonance, revealed that most of the vimentin tail region is disordered and have a higher degree of flexibility compared to the head and rod domains of the vimentin protein [27]. The indication of a flexible tail of the vimentin protein is supported by other studies $[28,29]$. Furthermore, 


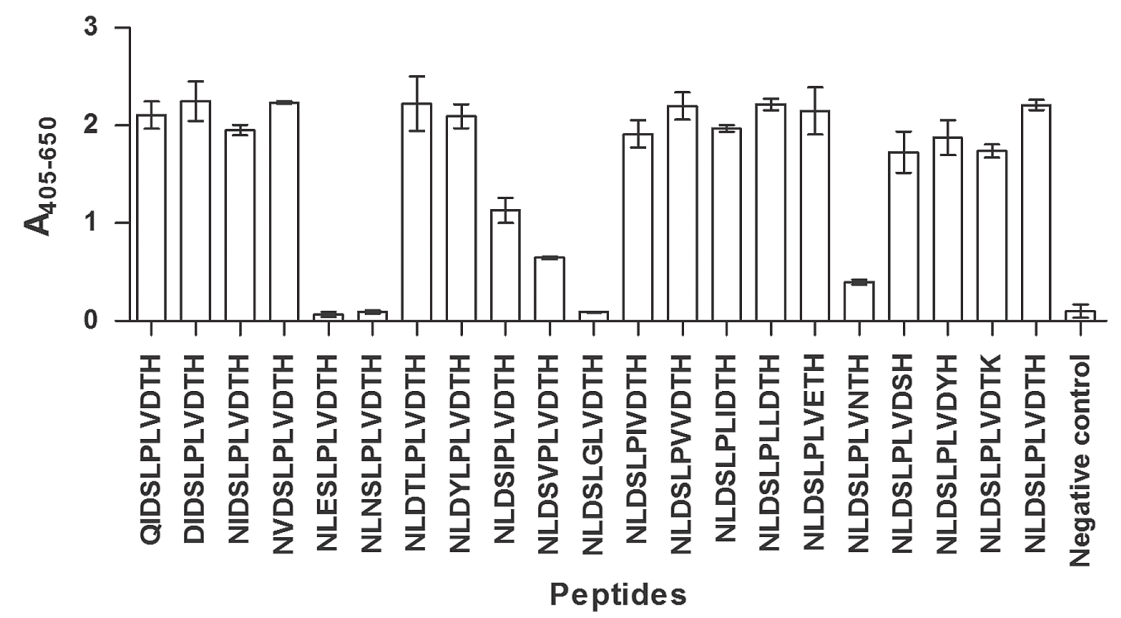

Figure 6. Reactivity of the vimentin antibody to substituted resin-bound vimentin peptides analyzed by modified ELISA. The peptide NLDSLPLVDTH was used as the control peptide with no substitutions. Uncoated resin was used as a negative control. Antibody reactivity to each peptide was analyzed in duplicate.

computer models have been used for predicting the secondary structure of the vimentin tail and they suggested extended strand and random coil [27,28]. These studies indicate that the identified vimentin epitope is found in a flexible region and this is a typical feature of antibody epitopes [6].

The epitope of the vimentin antibody was further characterized by Ala scanning and functionality scanning of the peptide NLDSLPLVDTH. As illustrated, the two negatively charged Asp3 and Asp9 residues were important for antibody binding (Figures 5 and 6). It was not possible to substitute Asp3 with Ala, Glu (similar functional group) or Asn (similar size), without notable influence on antibody reactivity, indicating a direct interaction between the Asp side chain and the antibody. These findings indicate that even small changes in this position disrupt the interaction between the epitope and the antibody, making this residue essential for antibody reactivity. In contrast, the Asp9 residue could be replaced with Glu without affecting the antibody reactivity, which may suggest that only the negative charge and not the specific side chain of Asp is important for reactivity in this position. The results obtained are in accordance with previous studies, showing that charged residues are important for antibody reactivity $[6,24,30]$. Furthermore, the results confirmed that the $C$-terminal Asp residue of the minimal epitope is important for antibody recognition. The two Asp residues were found to be the most important amino acids of the epitope for antibody reactivity and they may form ionic interactions and hydrogen bonds with the antibody.

In addition, the two hydrophobic Leu2 and Leu 5 residues were also shown to be important for antibody binding, as seen by a notable reduction in antibody reactivity upon Ala substitution. In contrast to the results of the Ala scanning, substitution of Leu2 with Ile or Val did not influence antibody reactivity, suggesting that the presence of an amino acid with an aliphatic side chain similar to the one in Leu is sufficient to retain antibody reactivity. The side chain of Ala is shorter than that of Leu and Ala was not sufficient to retain antibody reactivity as seen in the Ala scanning. Substitution of Leu 5 with Ile or Val resulted in a large reduction in antibody reactivity as seen in Figure 6, indicating the importance of the specific Leu side chain in this position. The Leu residues may contribute with van der Waals interactions. The importance of Leu residues for antibody reactivity was also confirmed by other studies $[10,13]$.

Moreover, substitution of Pro6 with Gly resulted in a very large reduction in antibody reactivity. Gly is a very flexible amino acid in contrast to Pro, which is a very rigid amino acid. This result may indicate that the Pro residue is important for the structure of the peptide, by constraining the peptide in a rather inflexible structure, since antibody reactivity was not reduced upon substitution with Ala which is not as flexible as Gly. The presence of Pro may indicate that the epitope is located in a $\beta$-turn since Pro is often seen in $\beta$-turns. This is in agreement with $\beta$-turns being the predominant motif in peptide-antibody complexes $[4,31]$.

Substitution of the remaining amino acids with various amino acids of similar or dissimilar side chain functionality including Ala showed nearly no reduction in antibody reactivity, suggesting that the side chains of these amino acids are not involved in direct interactions with the antibody. However, these amino acids may contribute to the conformation of the peptide which is important for proper antigen presentation or they may contribute with backbone interactions to the antibody.

In this epitope mapping study, we demonstrated that screening overlapping synthetic resin-bound peptides, covering a whole protein, with a monoclonal antibody is an efficient method for identification and mapping of epitopes in antigens targeted 
by antibodies. Determination of epitopes and characterization of the antibody-antigen interaction are relevant for diagnostics and treatment of several diseases as well as development of vaccines [16,32]. Moreover, discovery of new antigenic targets using epitope mapping may aid in understanding of the pathophysiology of different diseases, e.g. autoimmune diseases involving autoantibodies $[7,33,34]$.

To sum up, in this study only a single peptide was recognized in a specific manner by the vimentin antibody and the minimal and the complete epitope were determined to be the amino acid sequences LDSLPLVD and LDSLPLVDTH, respectively. Ala scanning and functionality scanning suggested that the two charged Asp residues were the amino acids most essential for antibody binding, underlining the importance of charged amino acids in epitopes.

The screening approach applied in this study is primarily based on detection of linear epitopes, due to practical limitaions, e.g. peptide length. It is unlikely that this type of assay could be applied for detection of conformational epitopes with great success. However, by applying longer peptides and to pool resin-bound peptides within wells, it may be possible to determine antigenicity within selected regions, e.g. domains. Thus when applying approaches like this in studying biomarkers analyzed by immunochemical methods, it only relates to antibodies detecting linear epitopes.

Declaration of interest: The authors report no conflict of interest. The authors alone are responsible for the content and writing of the paper.

\section{References}

[1] Mian IS, Bradwell AR, Olson AJ. Structure, function and properties of antibody binding sites. J Mol Biol 1991;217: 133-51.

[2] Kringelum JV, Nielsen M, Padkjær SB, Lund O. Structural analysis of B-cell epitopes in antibody:protein complexes. Mol Immunol 2013;53:24-34.

[3] Van Regenmortel MHV. What is a B-cell epitope? Methods Mol Biol 2009;524:3-20.

[4] Stanfield RL, Wilson IA. Protein-peptide interactions. Curr Opin Struct Biol 1995;5:103-13.

[5] Trier NH, Leth ML, Hansen PR, Houen G. Cross-reactivity of a human $\operatorname{IgG}(1)$ anticitrullinated fibrinogen monoclonal antibody to a citrullinated profilaggrin peptide. Protein Sci 2012;21:1929-41.

[6] Rubinstein ND, Mayrose I, Halperin D, Yekutieli D, Gershoni JM, Pupko T. Computational characterization of B-cell epitopes. Mol Immunol 2008;45:3477-89.

[7] Mahler M, Blüthner M, Pollard KM. Advances in B-cell epitope analysis of autoantigens in connective tissue diseases. Clin Immunol 2003;107:65-79.

[8] Sun J, Xu T, Wang S, Li G, Wu D, Cao Z. Does difference exist between epitope and non-epitope residues? Immunome res 201;7:1-11.

[9] Gershoni JM, Roitburd-Berman A, Siman-Tov DD, Tarnovitski Freund N, Weiss Y. Epitope mapping: the first step in developing epitope-based vaccines. BioDrugs 2007; 21:145-56.
[10] Geysen HM, Meloen RH, Barteling SJ. Use of peptide synthesis to probe viral antigens for epitopes to a resolution of a single amino acid. Proc Natl Acad Sci USA 1984; 81:3998-4002.

[11] Routsias JG, Tzioufas AG, Moutsopoulos HM. The clinical value of intracellular autoantigens B-cell epitopes in systemic rheumatic diseases. Clin Chim Acta 2004;340:1-25.

[12] Paterson Y. Delineation and conformational analysis of two synthetic peptide models of antigenic sites on rodent cytochrome c. Biochemistry 1985;24:1048-55.

[13] Tian Y, Ramesh CV, Ma X, Naqvi S, Patel T, Cenizal T, Tiscione M, Diaz K, Crea T, Arnold E, Arnold GF, Taylor JW. Structure-affinity relationships in the gp41 ELDKWA epitope for the HIV-1 neutralizing monoclonal antibody 2F5: effects of side-chain and backbone modifications and conformational constraints. J Peptide Res 2002; 59:264-76.

[14] Trier NH, Hansen PR, Vedeler CA, Somnier FE, Houen G. Identification of continuous epitopes of $\mathrm{HuD}$ antibodies related to paraneoplastic diseases/small cell lung cancer. J Neuroimmunol 2012;243:25-33.

[15] Trier NH, Hansen PR, Houen G. Production and characterization of peptide antibodies. Methods 2012;56:136-44.

[16] Irving MB, Pan O, Scott JK. Random-peptide libraries and antigen-fragment libraries for epitope mapping and the development of vaccines and diagnostics. Curr Opin Chem Biol 2001;5:314-24.

[17] Fidlerová H, Sovová V, Krekule I, Viklický V, Levan G. Immunofluorescence detection of the vimentin epitope in chromatin structures of cell nuclei and chromosomes. Hereditas 1992;117:265-73.

[18] Kouklis PD, Traub P, Georgatos SD. Involvement of the consensus sequence motif at coil $2 \mathrm{~b}$ in the assembly and stability of vimentin filaments. J Cell Sci 1992;102:31-41.

[19] Mor-Vaknin N, Punturieri A, Sitwala K, Markovitz DM. Vimentin is secreted by activated macrophages. Nature Cell Biol 2003;5:59-63.

[20] Pixley SK, Kobayashi Y, de Vellis J. A monoclonal antibody against vimentin: characterization. Brain Res 1984;317: 185-99.

[21] Osung OA, Chandra M, Holborow EJ. Intermediate filaments in synovial lining cells in rheumatoid arthritis and other arthritides are of vimentin type. Ann Rheum Dis 1982; 41:74-7.

[22] Vossenaar E, Després N, Lapointe E, van der Heijden A, Lora M, Senshu T, van Venrooij WJ, Menard HA. Rheumatoid arthritis specific anti-Sa antibodies target citrullinated vimentin. Arthritis Res Ther 2004;6:R142-50.

[23] Bang H, Egerer K, Gauliard A, Lüthke K, Rudolph PE, Fredenhagen G, Berg W, Feist E, Burmester G-R. Mutation and citrullination modifies vimentin to a novel autoantigen for rheumatoid arthritis. Arthritis Rheum 2007;56:2503-11.

[24] Petersen NH, Hansen PR, Houen G. Fast and efficient characterization of an anti-gliadin monoclonal antibody epitope related to celiac disease using resin-bound peptides. J Immunol Methods 2011;365:174-82.

[25] Wiik AS, Hoeier-Madsen M, Forslid J, Charles P, Meyrowitsch J. Antinuclear antibodies: a contemporary nomenclature using HEp-2 cells. J Autoimmun 2010;35:276-90.

[26] Price AS, Keil LB, DeBari VA. Immunocytochemical localization of cytoskeletal antigens in KB and HEp-2 cells. Diagn Clin Immunol 1988;5:400-13.

[27] Hess JF, Budamagunta MS, Aziz A, FitzGerald PG, Voss JC. Electron paramagnetic resonance analysis of the vimentin tail domain reveals points of order in a largely disordered region and conformational adaptation upon filament assembly. Protein Sci 2013;22:47-55.

[28] Strelkov SV, Herrmann H, Geisler N, Wedig T, Zimbelmann R, Aebi U, Burkhard P. Conserved segments $1 \mathrm{~A}$ and $2 \mathrm{~B}$ of the intermediate filament dimer: their atomic structures and role in filament assembly. EMBO J 2002;21:1255-66. 
[29] Chernyatina A, Nicolet S, Aebi U, Herrmann H, Strelkov SV. Atomic structure of the vimentin central $\alpha$-helical domain and its implications for intermediate filament assembly. Proc Natl Acad Sci USA 2012;109:13620-5.

[30] Amrutkar SD, Trier NH, Hansen PR, Houen G. Fine mapping of a monoclonal antibody to the N-Methyl D-aspartate receptor reveals a short linear epitope. Biopolymers 2012; 98:567-75.

[31] Trevino S, Schaefer S, Scholtz J, Pace C. Increasing protein conformational stability by optimizing b-turn sequence. J Mol Biol 2007;373:211-8.

\section{Supplementary material available online}

Supplementary Appendix.
[32] Timmerman P, Puijk WC, Meloen RH. Functional reconstruction and synthetic mimicry of a conformational epitope using CLIPS technology. J Mol Recognit 2007;5: 283-99.

[33] Sivalingam GN, Shepherd AJ. An analysis of B-cell epitope discontinuity. Mol Immunol 2012;51:304-9.

[34] Sebbag M, Moinard N, Auger I, Clavel C, Arnaud J, Nogueira L, Roudier J, Serre G. Epitopes of human fibrin recognized by the rheumatoid arthritis-specific autoantibodies to citrullinated proteins. Eur J Immunol 2006;36:2250-63. 